\title{
Graphene Defect Editing, Deposition, and Growth via E-Beam-Induced Organic Reactions in Aberration Corrected STEM
}

\author{
Ondrej Dyck, ${ }^{1,2}$ Songkil Kim, ${ }^{3}$ Sergei V. Kalinin ${ }^{1,2}$ and Stephen Jesse ${ }^{1,2}$ \\ 1. Center for Nanophase Materials Science, Oak Ridge National Laboratory, Oak Ridge, TN, \\ USA \\ 2. Institute for Functional Imaging of Materials, Oak Ridge National Laboratory, Oak Ridge, TN, \\ USA \\ 3. Department of Mechanical Engineering, Pusan National University, Republic of Korea
}

The development of nanotechnology relies critically upon the ability to manipulate fabrication parameters such that near atomic level material control becomes possible. With the rise of aberration correction in modern electron microscopy and the readily available, technologically relevant 2D materials, new possibilities are opening up in the realm of in situ scanning transmission electron microscopy (STEM).[1-4] Specifically, sub-angstrom probes are routinely achieved and in 2D materials these focused beams may be directed onto single atoms, thus creating an atomically localized fabrication environment which can be monitored in real time with atomic resolution.[57] Beam-induced sample changes are nothing new but the view that such alterations need to be strictly avoided is beginning to change. Here, we begin exploring the fabrication parameters needed to bring about controlled defect modification, deposition, and growth of graphene on graphene, including ex situ sample treatment and in situ temperature and feedback parameters.

Figure $1 \mathrm{a}$ )-c) details the chemical modification of a graphene lattice where a defect cluster of Si dopants is removed from the lattice and replaced with $\mathrm{C}$ through a combination of electron beam ionization of hydrocarbon gas surrounding the sample and subsequent chemical reaction with and removal of the Si defects at room temperature. This alteration occurred under the same sample conditions during which single and multilayer graphene growth was possible, d)-g). Through continuous scanning, a $\sim 3 \mathrm{~nm}$ hole in the graphene lattice was healed (which also acted to incorporate as substitutional dopants the $\mathrm{Si}$ atoms attached to the edge of the hole), and, at the top of the images, we see double, triple, and quadruple layers of graphene/graphite growing. Because we do not observe spontaneous deposition anywhere else on the surface, it is clear there is a strong preference for adatom migration along the surface and attachment to step edges. This is in contrast to the more rapid deposition often observed in graphene samples brought on by the outgassing of residual polymeric materials and solvents used in sample preparation. Figure $1 \mathrm{~h}$ ) illustrates this type of heavy deposition where primarily amorphous carbon is observed. Nevertheless, heating the amorphous deposition results in conversion to graphite, $\mathrm{j}$ ) and $\mathrm{k}$ ). This suggests that e-beam patterned conductive graphitic nanowires and resistive amorphous $\mathrm{C}$ can be patterned at the nanometer scale in situ in the STEM.

In an attempt to gain greater control over the growth, a customized beam control and feedback (real-time analysis) hardware/software interface with the STEM was developed. In this application the beam was scanned back and forth, the intensity analyzed, and the beam advanced automatically when crossing an adjustable threshold. The sample was held at a variety of temperatures, 600, 650, 700 , and $750{ }^{\circ} \mathrm{C}$. Graphitic growth was achieved at each temperature without significant differences between the results, 1). The inset in 1) shows a magnified view of the $650{ }^{\circ} \mathrm{C}$ deposition 
(scale bar $1 \mathrm{~nm}$ ). In addition, i) shows a typical image of the graphene layers acquired under these conditions. The streaks indicate the significant influx and rearrangement of $\mathrm{C}$ atoms attaching to the step edges. In agreement with d)-g), no deposition directly onto the graphene lattice was observed. Deposition had to be initiated at a defect or the edge of another layer. However, direct deposition was possible under the more heavily contaminating conditions shown in $\mathrm{h}$ ), therefore, one could imagine depositing a starting seed and changing the fabrication parameters to continue the nanowire growth. Similar chemical alterations, deposition and growth are likely possible on other 2D materials as well and may lead to the ability to dope h-BN with $\mathrm{C}$ on the nanometer scale or grow conductive nanowires along the surface of dichalcogenides.
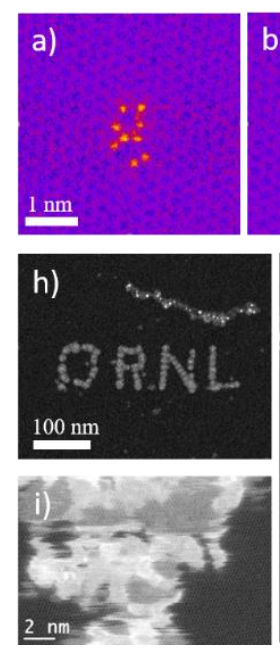
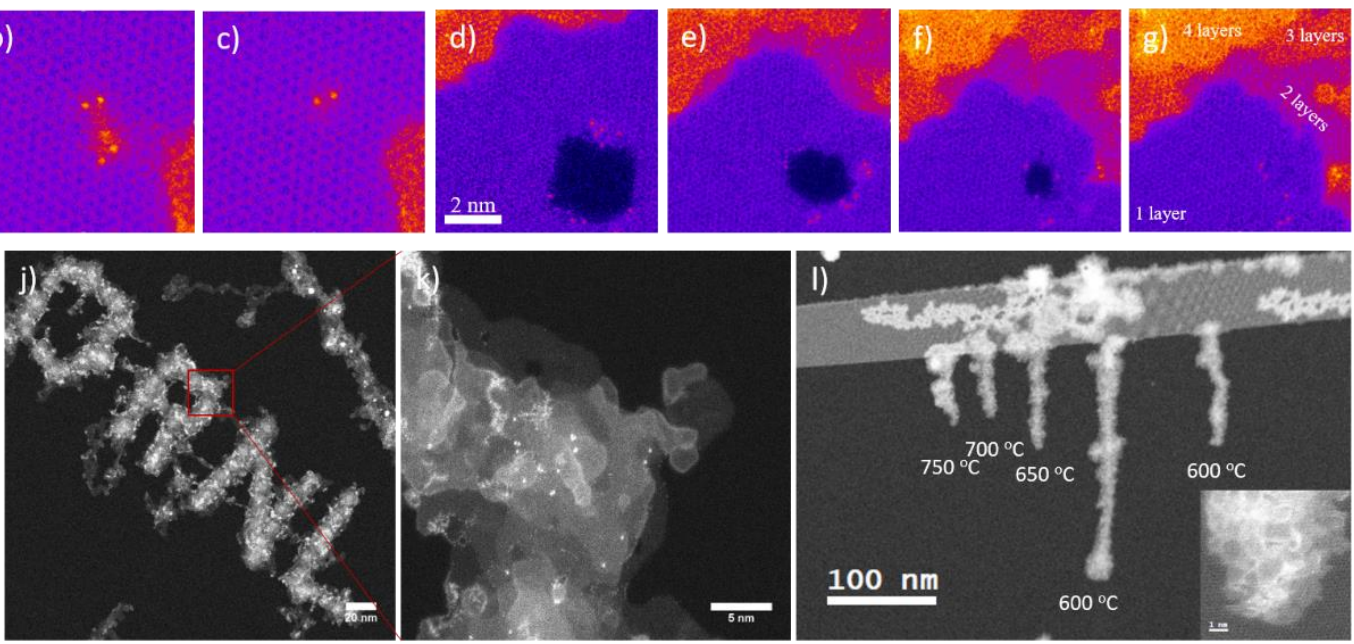

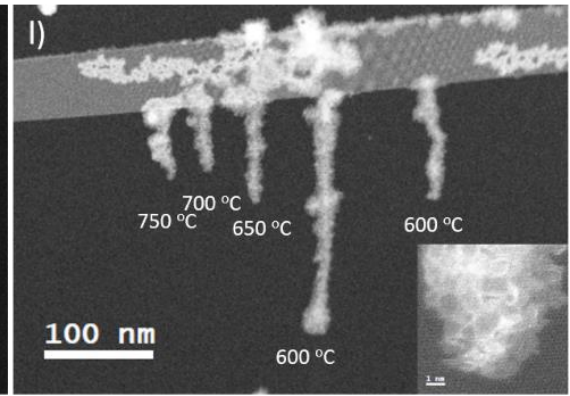

Figure 1 a)-c) False color images of electron beam driven chemical reactions where $\mathrm{C}$ from hydrocarbon gas is ionized and reacts with a Si dopant cluster. The $\mathrm{Si}$ atoms are removed from the lattice and it is restored to defect free graphene. In a similar way, in d)-g) we observe the growth of 1,2,3, and 4-layer graphene through the attachment of the carbon gas/adatoms to the graphene edges (images in false color). h), j), and k) illustrate amorphous deposition, h), and transition from amorphous to graphitic carbon upon heating, j) and k). 1) shows the controlled growth of graphite nanowires at various temperatures using a custom beam control and feedback interface. The inset in 1) shows a magnified view of the edge of the $650{ }^{\circ} \mathrm{C}$ growth (scale bar is $1 \mathrm{~nm}$ ). i) shows a typical image acquired under these conditions where the streaks along the edges indicate significant adatom aggregation and restructuring along the edges of the graphite layers.

[1] Jiang, N., et al., MRS Bulletin. 42(2017), p. 653-659.

[2] Kalinin, S.V. and S.J. Pennycook, MRS Bulletin. 42(2017), p. 637-643.

[3] Mishra, R., et al., MRS Bulletin. 42(2017), p. 644-652.

[4] Zhao, X., et al., MRS Bulletin. 42(2017), p. 667-676.

[5] Dyck, O., et al., Applied Physics Letters. 111(2017), p. 113104.

[6] Susi, T., et al., 2D Materials. 4(2017), p. 042004.

[7] Susi, T., et al., Physical Review Letters. 113(2014), p. 115501. 